\title{
Late cardiopulmonary and musculoskeletal exercise performance after repair for total anomalous pulmonary venous connection during infancy
}

Michael G. McBride, PhD, ${ }^{a}$ Paul M. Kirshbom, MD, ${ }^{f}$ J. William Gaynor, MD, ${ }^{b}$ Richard F. Ittenbach, PhD, ${ }^{c}$

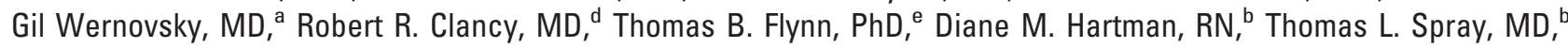
Ronn E. Tanel, MD, ${ }^{a}$ Mayra C. Santiago, $\mathrm{PhD}^{\mathrm{g}}{ }^{\mathrm{a}}$ and Stephen M. Paridon, $\mathrm{MD}^{\mathrm{a}}$

Supplemental material is available online.
From the Divisions of Cardiology ${ }^{\mathrm{a}}$ Cardiothoracic Surgery, ${ }^{\mathrm{b}}$ Biostatistics and Epidemiology, ${ }^{\mathrm{c}}$ Neurology, ${ }^{\mathrm{d}}$ and Psychology, ${ }^{\mathrm{e}}$ The Children's Hospital of Philadelphia and the University of Pennsylvania School of Medicine, Philadelphia, Pa; the Division of Cardiothoracic Surgery, ${ }^{\mathrm{f}}$ Emory University, Atlanta, Ga; and Department of Kinesiology, ${ }^{\mathrm{g}}$ Temple University, Philadelphia, $\mathrm{Pa}$.

Supported in part by a grant from the National Institutes of Health General Clinical Research Center (Grant No. M01-RR-00240).

Received for publication Oct 18, 2006; accepted for publication Dec 13, 2006.

Address for reprints: Michael G. McBride, $\mathrm{PhD}$, Division of Cardiology, Children's Hospital of Philadelphia, 34th and Civic Center Blvd, Philadelphia, PA 19104 (E-mail: mcbride@email.chop.edu).

J Thorac Cardiovasc Surg 2007;133:1533-9

$0022-5223 / \$ 32.00$

Copyright $(92007$ by The American Association for Thoracic Surgery

doi:10.1016/j.jtcvs.2006.12.032
Objectives: We evaluated cardiopulmonary function at rest and during exercise in children after surgical repair for total anomalous pulmonary venous connection.

Background: Long-term assessment of cardiopulmonary function during exercise in children after repair for total anomalous pulmonary venous connection during infancy is limited.

Methods: Resting lung function and cardiopulmonary function during maximal ramp cycle ergometry were evaluated in 27 patients (age $=11 \pm 4$ years, 20 were male). Peak oxygen consumption, ventilatory anaerobic threshold, and physical working capacity were compared with normal reference values. Neurologic assessment included neuromuscular function, inattentiveness, and hyperactivity. Patientand procedure-related variables were assessed for association with peak oxygen consumption, ventilatory anaerobic threshold, and physical working capacity.

Results: Compared with healthy children, peak oxygen consumption $(88 \% \pm 16 \%$ of predicted) and ventilatory anaerobic threshold (91\% $\pm 21 \%$ of predicted) were mildly reduced. Chronotropic impairment was observed in 7 patients (32\%). Patients with impaired resting lung mechanics were more likely to have impairment in peak oxygen consumption $(P<.05)$. Breathing reserve was normal. Specific anatomy and all operative factors did not have a significant impact on overall exercise performance. Composite score for fine and gross motor function was associated with lower ventilatory anaerobic threshold $(P<.05)$.

Conclusions: Exercise performance is mildly impaired at long-term follow-up after total anomalous pulmonary venous connection repair during infancy. Residual pulmonary abnormalities are common and associated with lower exercise performance. Neurologic abnormalities are evident in a subgroup, but the impact on late exercise performance is inconclusive.

$\mathrm{T}$ otal anomalous pulmonary venous connection (TAPVC) presents with several anatomic variants that subject patients to different risks and potential complications. Improvements in surgical techniques, preoperative stabilization, and postoperative care have resulted in lower mortality and increased shortand long-term survival over the past 30 years. ${ }^{1}$ However, the majority of studies report early surgical and long-term hemodynamic outcomes by cardiac catheterization or resting echocardiography. Assessment of cardiovascular, pulmonary, and musculoskeletal systems during exercise has been limited. Residual cardiopulmonary abnormalities undetected at rest may result in impaired function during exercise. ${ }^{2}$ Although these abnormalities seem to be well tolerated, no data exist that 


$$
\begin{aligned}
& \text { Abbreviations and Acronyms } \\
& \begin{aligned}
\mathrm{BR} & =\text { breathing reserve } \\
\mathrm{DS} / \mathrm{TV} & =\text { dead space-to-tidal volume ratio } \\
\mathrm{f} & =\text { respiratory rate } \\
\mathrm{FEV}_{1} & =\text { forced expiratory volume in } 1 \text { second } \\
\mathrm{FVC} & =\text { forced vital capacity } \\
\mathrm{MVV} & =\text { maximal voluntary ventilation } \\
\mathrm{O}_{2} \mathrm{P} & =\text { oxygen pulse } \\
\mathrm{PWC} & =\text { peak work capacity } \\
\mathrm{TAPVC} & =\text { total anomalous pulmonary venous } \\
& \text { connection } \\
\mathrm{TV} & =\text { tidal volume } \\
\mathrm{VAT} & =\text { ventilatory anaerobic threshold } \\
\mathrm{VE} & =\text { minute ventilation }
\end{aligned}
\end{aligned}
$$

describe the long-term impact of isolated TAPVC surgically repaired during infancy on indices of exercise performance.

The purpose of this study was to evaluate long-term cardiac, pulmonary, and musculoskeletal function at rest and during exercise in children after surgical repair for TAPVC during infancy, and to estimate the impact of perioperative and specific neurologic factors on long-term cardiopulmonary exercise performance.

\section{Materials and Methods \\ Patient Population}

Children eligible to participate in this study included all patients with simple TAPVC admitted to The Children's Hospital of Philadelphia between November 1983 and November 1996. Simple TAPVC is defined as TAPVC with no other cardiac anomalies except for patent foramen ovale, atrial septal defect, coarctation of the aorta, or patent ductus arteriosus. ${ }^{3} \mathrm{~A}$ total of 104 children were identified as candidates for inclusion in the study, 4 of whom died before surgical intervention; of this total, 100 survived to surgery. The hospital mortality rate was $14 \%$, and 2 children died long-term postoperatively. Ten patients were lost to follow-up. Of the 74 late survivors, 60 were surgically repaired during infancy and at least 6 years of age at the time of this study. The parents/guardians of 42 of these 60 children were successfully contacted. Of these 42 families, 27 agreed to participate in this study. Before testing, each patient and a parent/guardian signed an informed assent/consent in accordance with The Children's Hospital of Philadelphia Institutional Review Board, Committee for the Protection of Human Subjects.

\section{Resting Lung Mechanics}

Pulmonary function was evaluated before the exercise test using standard methods for spirometry, lung volumes, and conductance. ${ }^{4}$ Forced vital capacity (FVC), forced expiratory volume in 1 second $\left(\mathrm{FEV}_{1}\right)$, forced expiratory flows at $25 \%$ to $75 \%$ of $\mathrm{FVC}\left(\mathrm{FEF}_{25-75}\right)$, and the ratio $\mathrm{FEV}_{1} / \mathrm{FVC}$ were measured and compared with appropriate reference values. ${ }^{5}$ Maximal voluntary ventilation (MVV) was used to calculate the breathing reserve (BR) at peak exercise using the formula: $\mathrm{BR}=[1-(\mathrm{VE} / \mathrm{MVV})] \times 100$, where $\mathrm{VE}$ equals the minute ventilation (VE) at maximal exercise. ${ }^{6}$

\section{Cardiovascular Monitoring}

Cardiac rhythm was monitored continuously and recorded intermittently during each study using standard electrocardiography. A peak heart rate response less than 2 standard deviations from the normal value (heart rate $<185$ beats $/ \mathrm{min}$ ) was used as criteria for chronotropic impairment. ${ }^{7,8}$ Blood pressure was measured by auscultation at rest and every 3 minutes during exercise and recovery.

\section{Exercise Capacity}

Patients were exercised to maximal volition (respiratory exchange ratio $>1.10$ ) using a 1-minute incremental ramp cycle ergometer protocol. ${ }^{7}$ Metabolic and ventilatory data were obtained on a breath-by-breath basis using a metabolic cart (SensorMedics, Yorba Linda, Calif). Parameters measured included minute oxygen consumption $\left(\mathrm{VO}_{2}\right)$, minute carbon dioxide production $\left(\mathrm{VCO}_{2}\right)$, $\mathrm{VE}$, respiratory exchange ratio, respiratory rate (f), tidal volume (TV), dead space-to-tidal volume ratio (DS/TV), and the ventilatory equivalents for oxygen and carbon dioxide $\left(\mathrm{VE} / \mathrm{VO}_{2}, \mathrm{VE} /\right.$ $\left.\mathrm{VCO}_{2}\right)$. The oxygen pulse $\left(\mathrm{O}_{2} \mathrm{P}\right)$ for each patient was calculated by dividing the $\mathrm{VO}_{2}$ by the simultaneous measured heart rate and indexed to height (centimeters). ${ }^{9}$ The ventilatory anaerobic threshold (VAT) was measured by the V-slope method. ${ }^{10}$ Ventilatory efficiency during exercise was assessed by calculation of the slope of the increase in VE versus $\mathrm{VCO}_{2}$ from the onset of workup to the inflection point in the V-slope. Mechanical work efficiency was determined by calculating the change in work rate, measured in Watts $(\mathrm{W})$, over the change in oxygen consumption $\left(\Delta \mathrm{W} / \Delta \mathrm{VO}_{2}\right)$ measured at an respiratory exchange ratio of 0.95 or less. ${ }^{11}$ Metabolic and ventilatory data were compared with healthy age- and gender-matched children using the same exercise protocol. ${ }^{9,12}$

\section{Echocardiography}

Resting echocardiograms included a complete 2-dimensional echocardiogram with Doppler evaluation and were recorded on videotape and/or digital transfer medium. Echocardiography assessed the presence of a residual atrial septal defect and the presence of obstruction within the pulmonary veins. Specific measurements during the echocardiogram included right ventricular pressure estimate, when observable from the tricuspid insufficiency jets, and left ventricular shortening fraction.

\section{Neurologic Examination}

The developmental history and neurologic examinations were conducted by a single, senior, board-certified pediatric neurologist who is familiar with the neurodevelopmental issues in children who have undergone infant heart surgery. The protocol for the neurologic examination has been described. ${ }^{13}$ Briefly, the examination assessed muscle tone, strength, symmetry, reflexes, and coordination of fine and gross motor activities. All findings are recorded on case report forms. Abnormal motor abnormalities included those with a diagnosis of cerebral palsy or obvious impairments of motor function. Suspect scores were coded for those with more subtle disturbances, such as impaired hand or finger-tapping sequences, uncoordinated rapid alternating movements of the hands, or inability to hop repetitively on 1 foot. The composite score reflected any abnormality of fine or gross motor ability. The impression of hyperactivity or attention deficit was 
TABLE 1. Clinical, anatomic, and perioperative data of subjects

\begin{tabular}{lc}
\hline Variable & Value \\
\hline Demographics & \\
Male, $\mathrm{n}(\%)$ & $20(74)$ \\
Female, $\mathrm{n}(\%)$ & $7(26)$ \\
Gestation, w & $38.8 \pm 2.5$ \\
Birth weight, g & $3289.5 \pm 713.3$ \\
Age at surgery, d & $36.7 \pm 44.7$ \\
Anatomy & \\
Supracardiac, $\mathrm{n}(\%)$ & $14(52)$ \\
Intracardiac, $\mathrm{n}(\%)$ & $3(11)$ \\
Infracardiac, $\mathrm{n}(\%)$ & $10(37)$ \\
Pulmonary venous obstruction, $\mathrm{n}(\%)$ & $5(19)$ \\
Perioperative data & \\
Intubated preoperatively, $\mathrm{n}(\%)$ & $7(26)^{*} \dagger$ \\
Cardiopulmonary bypass, min & $70.7 \pm 14.3$ \\
Circulatory arrest, min & $34.8 \pm 10.2$ \\
ICU length of stay, d & $11.6 \pm 11.3$ \\
Length of hospital stay, d & $16.5 \pm 11.7$ \\
Need for reintervention, $\mathrm{n}(\%)$ & $6(22)$ \\
\hline
\end{tabular}

$I C U$, Intensive care unit. Values represent mean \pm standard deviation, or number of observations $(\mathrm{n})$ and percentage of total population (\%). Length of hospital stay: surgery to discharge. *Significantly younger age, $P<.01$. tSignificantly longer period of ICU length of stay, $P<.01$.

based on parental interview and clinical observation but not by a formal psychologic battery.

\section{Data Analysis}

Data analysis consisted of generating descriptive statistics for all relevant variables in the data set. Independent $t$ tests were used to detect differences between the patients who underwent TAPVC and age and gender-match reference data in 4 key areas: (1) cardiovascular function at rest, (2) cardiovascular function during exercise, (3) pulmonary function at rest, and (4) pulmonary function during exercise. Single- and multiple-covariate linear regression models were fitted and tested for 3 exercise-related end points, $\mathrm{VO}_{2}$ peak, VAT, and peak work capacity (PWC). Any single covariate with a $P$ value less than .15 was considered a candidate for inclusion in a multiple-covariate equation. Because of the exploratory nature of the study, and the general lack of exerciserelated information on school-age survivors of TAPVC, alpha was not adjusted for multiple comparisons, thereby keeping the criterion for statistical significance at $\alpha=0.05$ level for all analyses.

\section{Results}

Patient demographics and anatomy of the pulmonary venous drainage are summarized in Table 1. All patients were clinically healthy with none taking routine cardiac medications.

Perioperative data are also summarized in Table 1. All patients had a period of deep hypothermic circulatory arrest during their surgical procedure. Patients who required preoperative intubations $(n=7)$ were significantly younger
TABLE 2. Cardiovascular and metabolic responses during exercise

\begin{tabular}{lcc}
\hline Variable & Value $^{*} \dagger$ & Percentage of predicted \\
\hline $\mathrm{HR}, \mathrm{beats} / \mathrm{min}$ & $184.4 \pm 14.3^{*} \ddagger$ & $92 \pm 9$ \\
$\mathrm{VO}_{2}, \mathrm{~mL} / \mathrm{kg} / \mathrm{min}$ & $36.3 \pm 6.9^{*} \S$ & $88 \pm 16$ \\
$\mathrm{VO}_{2}, \mathrm{~mL} / \mathrm{min}$ & $1448.3 \pm 527.3^{*} \S$ & $88 \pm 16$ \\
$\mathrm{VCO}_{2}, \mathrm{~mL} / \mathrm{min}$ & $1780.1 \pm 668.2^{*}$ & - \\
$\mathrm{O}_{2} \mathrm{P}, \mathrm{mL} / \mathrm{beat}$ & $7.8 \pm 2.9^{*}$ & $96 \pm 16$ \\
$\mathrm{PWC}, \mathrm{W}$ & $128.7 \pm 52.2^{*}$ & $104 \pm 21$ \\
$\mathrm{VAT}, \mathrm{mL} / \mathrm{kg} / \mathrm{min}$ & $22.1 \pm 5.4 \dagger \ddagger$ & $91 \pm 21$ \\
$\mathrm{VAT}, \mathrm{mL} / \mathrm{min}$ & $868.1 \pm 37.2 \dagger \ddagger$ & $91 \pm 21$ \\
VAT, $\%$ of & $63.2 \pm 9.1 \dagger$ & - \\
VO & \\
Weak & & \\
Work efficiency, $\%$ & $27.3 \pm 7.2 \dagger$ & $95 \pm 26$ \\
RER & $1.2 \pm 0.1^{*}$ &
\end{tabular}

$\mathrm{HR}$, Heart rate; $\mathrm{VO}_{2}$, minute oxygen consumption; $\mathrm{VCO}_{2}$, minute carbon dioxide production; $\mathrm{O}_{2} P$, oxygen pulse; $P W C$, physical working capacity; $V A T$, ventilatory anaerobic threshold; $R E R$, respiratory exchange ratio. Values are represented as mean \pm standard deviation and percentage of predicted for age and gender. ${ }^{*}$ Peak exercise $(n=22)$. TSubmaximal exercise $(\mathrm{n}=25) . \ddagger P<.05$ for age and gender. $§ P<.01$ for age and gender.

than those who were not intubated $(9.3 \pm 5.1$ days vs $34.0 \pm$ 46.2 days, $P=.01$ ), and they required significantly longer postoperative intensive care unit stays $(18.3 \pm 16.1$ days vs $8.1 \pm 5.0$ days, $P<.01)$. Patients with evidence of pulmonary venous obstruction at presentation $(\mathrm{n}=5)$ tended to require preoperative intubation (4/5 [80\%] vs 3/22 [14\%], $P$ $=.05$ ). Six patients (22\%) required late surgical reintervention (14.7 \pm 18.8 months) consisting of complete revision of TAPVC repair $(n=5)$ or closure of residual septal defect $(n=1)$. A comparison between the patients who voluntarily enrolled and those who were eligible but did not return for study participation demonstrated no significant differences between the groups for the demographic and perioperative variables listed in Table $1 .^{14}$

Demographic and echocardiographic data at the time of testing are summarized in Table E1. No patient had greater than mild tricuspid regurgitation on resting echocardiography. There was evidence of mild flow acceleration at the pulmonary venous anastomosis in 1 patient whose right ventricular pressure estimate was $28 \mathrm{~mm} \mathrm{Hg}$.

\section{Cardiovascular Function}

Cardiovascular and metabolic data during exercise are summarized in Table 2. On the basis of metabolic and ventilatory data, 22 patients $(81 \%)$ were thought to have achieved peak aerobic capacity during testing. However, VAT was measurable in 3 of 5 patients who did not achieve peak aerobic capacity and were included in the analysis of submaximal exercise performance.

Evaluation of electrocardiographic responses to maximal exercise showed all but 5 patients (19\%) were in sinus 
TABLE 3. Indices of resting lung mechanics

\begin{tabular}{lc}
\hline Variable & Value \\
\hline FVC, $\mathrm{L}$ & $2.37 \pm 0.92^{*}$ \\
$\mathrm{FVC}, \%$ of predicted & $89 \pm 15$ \\
$\mathrm{FEV}_{1}, \mathrm{~L}$ & $2.06 \pm 0.77^{*}$ \\
$\mathrm{FEV}_{1}, \%$ of predicted & $87 \pm 14$ \\
$\mathrm{FEV}_{1} / \mathrm{FVC}, \%$ & $85 \pm 7$ \\
$\mathrm{FEF}_{25-75}, \mathrm{~L}$ & $2.27 \pm 1.02^{*}$ \\
$\mathrm{FEF}_{25-75} \%$ of predicted & $78 \pm 24$
\end{tabular}

$F V C$, Forced vital capacity; $F E V_{1}$, forced expiratory volume in 1 second; $F E F_{25-75}$, forced expiratory flow at $25 \%$ to $75 \%$ of forced vital capacity. Values represent mean \pm standard deviation. ${ }^{*} P<.01$ for age, gender, and height.

rhythm throughout their exercise tests. Rare $(<1 \%)$ isolated ectopic atrial or ventricular complexes were observed in 4 patients $(15 \%)$. In the 22 patients who achieved peak aerobic capacity during the study, an attenuated heart rate response $(167.8 \pm 13.5$ beats/min) was observed in $7(32 \%)$.

Aerobic capacity, as measured by percentage of predicted $\mathrm{VO}_{2}$ peak, for the group was significantly lower than normal values for age and gender $(P=.01)$. However, only 3 patients $(13 \%)$ had significant impairment of aerobic capacity below the $95 \%$ confidence limit for age and gender. Raw VAT and percentage of predicted values, reported in 25 patients (93\%), was significantly lower than the normal value for age and gender $(P=.01)$. Mean raw VAT for 3 of the 5 patients who did not achieve peak aerobic capacity was $19.5 \pm 6.8 \mathrm{~mL} / \mathrm{kg} / \mathrm{min}(90 \% \pm 16 \%$ of predicted $)$.

\section{Resting Pulmonary Function}

Indices of resting lung mechanics are summarized in Table 3. FVC was significantly $(P=.01)$ below the mean predicted value for age and height in all but 5 patients $(18 \%)$. Similarly, significant differences were observed in $\mathrm{FEV}_{1}$ $(P=.01)$ and $\mathrm{FEF}_{25-75}(P=.01)$ in 4 patients $(15 \%)$ and 5 patients $(18 \%)$, respectively, when compared with normal values for age and height. When $\mathrm{FEV}_{1}$ was expressed as a percentage of the $\mathrm{FVC}\left(\mathrm{FEV}_{1} / \mathrm{FVC}\right)$, this ratio was within the expected value for all but 9 patients (33\%).

\section{Exercise Pulmonary Function}

Ventilatory parameters during exercise are summarized in Table 4. Resting ventilatory parameters were essentially unremarkable. However, estimated DS/TV at rest $(49.1 \% \pm$ $6.0 \%)$ was significantly above the mean for age and gender in all but 1 patient $(4 \%)(P=.01)$. Estimated DS/TV at peak exercise was also significantly above the mean in all but 5 patients (22\%) who achieved peak aerobic capacity $(P=.01)$. Although notable increases in mean values for " $\mathrm{f}$ " and TV were observed during submaximal exercise from resting values, a blunted increase in mean TV was noted at a point approximating the VAT $(62 \%$ of peak
TABLE 4. Ventilatory parameters during exercise

\begin{tabular}{|c|c|c|}
\hline Variable & Submaximal exercise* & $\overline{\text { Peak exerciset }}$ \\
\hline $\mathrm{f}$, breaths/min & $44.1 \pm 7.6$ & $58.8 \pm 6.7 \ddagger$ \\
\hline $\mathrm{TV}, \mathrm{mL} / \mathrm{min}$ & $896.3 \pm 323.2$ & $1080.1 \pm 420.1$ \\
\hline $\mathrm{VE}, \mathrm{L} / \mathrm{min}$ & $43.2 \pm 11.8$ & $68.4 \pm 22.0 \S$ \\
\hline $\mathrm{f} / \mathrm{TV}, \%$ & $48 \pm 21$ & $63 \pm 29$ \\
\hline TV/FVC, \% & $36 \pm 8$ & $41 \pm 16$ \\
\hline $\mathrm{VE} / \mathrm{VO}_{2}, \%$ & $44 \pm 47$ & $47 \pm 6$ \\
\hline VE/VCO $2, \%$ & $41 \pm 10 \ddagger$ & $41 \pm 6$ \\
\hline VE vs $\mathrm{VCO}_{2}$, slope & $43 \pm 25 \ddagger$ & \\
\hline DS/TV, $\%$ & $38 \pm 5$ & $29 \pm 7 \ddagger$ \\
\hline $\mathrm{BR}, \%$ & - & $19 \pm 11$ \\
\hline
\end{tabular}

$f$, Respiratory rate; $T V$, tidal volume; $V E$, minute ventilation; $F V C$, forced vital capacity; $V E / V O_{2}$, ventilatory equivalent for oxygen; $V E / V C O_{2}$, ventilatory equivalent for carbon dioxide; $R E R$, respiratory exchange ratio; $D S / T V$, dead space-to-tidal volume ratio; $B R$, breathing reserve. Values represent mean \pm standard deviation. ${ }^{*} \mathrm{n}=25 . \mathrm{tn}=22 . \ddagger P<.01$ for age and gender. $\S P<.05$ for age and gender.

exercise). Therefore, a larger fraction of VE was primarily driven by " $\mathrm{f}$ " at exercise intensities beyond the VAT. Those with high "f" at peak exercise were more likely to have high DS/TV at peak exercise $(P=.01)$.

BR at peak exercise for the group represented normal pulmonary reserve when compared with age- and gendermatched reference values. BR was less than $15 \%$ in 10 of the patients $(45 \%)$. This is the level below which pulmonary insufficiency potentially impairs exercise performance. Four of these 10 patients had normal values for $\mathrm{VO}_{2}$ peak.

\section{Neurologic Function}

The results from the neurologic examinations are presented in Table E2. Neurologic function in 10 of the 27 subjects (37\%) was rated as abnormal or suspect on the overall neurologic assessment. Forty-four percent of the subjects were found to have deficits in 1 or more of the 4 domains (attention, hyperactivity, fine motor, gross motor) assessed during this study. Neuromuscular assessment was rated as abnormal or suspect in 7 of the subjects $(26 \%)$.

\section{Factors Associated With Exercise Performance}

Eleven potential perioperative risk factors (Table 1) were tested for association to predict long-term exercise performance, as measured by percent of predicted values for 3 primary end points $\left(\mathrm{VO}_{2}\right.$ peak, VAT, and PWC). Anatomy of the pulmonary venous drainage and the presence of pulmonary venous obstruction on presentation, as well as all operative variables, did not have a significant impact on overall exercise performance as measured by $\mathrm{VO}_{2}$ peak, VAT, and PWC. Although the need for late reintervention was significantly associated with lower PWC $(P=.04)$, it was not associated with neuromuscular function $(P=.55)$ or work efficiency $(P=.38)$. 
Fourteen different cardiovascular and pulmonary risk factors (Tables E1 and 2-4) were also tested for association with long-term exercise performance. $\mathrm{O}_{2} \mathrm{P}$ at peak exercise was associated with higher values for $\mathrm{VO}_{2}$ peak $(P=.03)$ and PWC $(P=.02)$. There was no association between any candidate of exercise performance and the chronotropic response. Those patients with impaired $\mathrm{FEV}_{1}$ and $\mathrm{FEV}_{1} /$ $\mathrm{FVC}$ were more likely to have impairment in $\mathrm{VO}_{2}$ peak $(P=$ .04 and $P=.02$, respectively), although those patients who achieved higher VE during exercise were more likely to achieve greater $\mathrm{VO}_{2}$ peak $(P=.03)$. Although DS/TV at peak exercise was above the mean for age and gender, it was not associated with lower $\mathrm{VO}_{2}$ peak $(P=.63)$.

Six different neurologic risk factors (Table E2) were tested for inclusion in a multicovariate statistical model to predict long-term exercise performance. Whereas there was a tendency toward lower PWC $(P=.11)$ in those subjects who scored lower in the composite score of neuromuscular function (gross motor and fine motor), none of the assessments for neurologic function significantly affected overall exercise performance as measured by $\mathrm{VO}_{2}$ peak and PWC. However, lower composite score in neuromuscular function was significantly associated with lower VAT $(P=.03)$.

\section{Determinants of Aerobic Exercise Performance}

To test for synergistic effects, covariates with $P$ less than .15 were entered into multicovariate analyses to arrive at the best fitting model for each of the 3 primary outcomes of exercise performance (Table E3). According to multicovariate analysis using the subjects' demographics, perioperative data, and pulmonary variables, the strongest predictors of peak exercise performance, as measured by $\mathrm{VO}_{2}$ peak, were $\mathrm{FEV}_{1}$ and $\mathrm{O}_{2} \mathrm{P}(P=.02)$. The strongest predictors of submaximal exercise performance, as measured by VAT, were diastolic blood pressure and neuromuscular function $(P=.07)$. Last, the strongest predictors of musculoskeletal performance, as measured by $\mathrm{PWC}$, were gender and $\mathrm{O}_{2} \mathrm{P}$ $(P=.01)$.

\section{Discussion \\ Cardiovascular Function}

The findings from this study indicate aerobic exercise performance in children surgically repaired for TAPVC during infancy is mildly impaired compared with exercise performance in healthy children. However, a majority (86\%) of these children have $\mathrm{VO}_{2}$ peak values within the $95 \%$ confidence limit for age and gender. This is a modest improvement from the earlier report by Paridon and colleagues, ${ }^{2}$ in which the aerobic capacity was $82 \%$ of predicted for age and gender in 6 children surgically repaired for TAPVC during infancy.

Similarly, data from this study suggest mild impairment in submaximal exercise performance, as measured by VAT.
Although less impaired than $\mathrm{VO}_{2}$ peak, the mild impairment in submaximal exercise performance may be more discouraging than that observed in $\mathrm{VO}_{2}$ peak. The VAT is often considered a more reliable marker of aerobic fitness because of its ability to be sustained over a longer period of time and its sensitivity to aerobic deconditioning and sedentary lifestyle. $^{6}$

Our data indicate that PWC and work efficiency is within normal range for age and gender despite limited aerobic parameters of performance and is superior to that reported in other cardiac lesions requiring early repair. ${ }^{8,15}$ This is not surprising given that lesions requiring early surgical repair, such as tetralogy of Fallot and atrial switch physiology, often have residual hemodynamic abnormalities.

The incidence of mild chronotropic impairment seen in this study is inconsistent with the findings of previous studies. An early report by Byrum and colleagues ${ }^{16}$ found normal sinus node function in 7 late survivors, whereas a more recent study by Paridon and colleagues ${ }^{2}$ found mild chronotropic incompetence in 5 of 6 children at late followup. Reasons for these discrepancies are unclear and probably multifactorial. However, it is not uncommon for children with cardiac lesions requiring extensive atrial surgery to exhibit some level of sinus node dysfunction. Often a blunted chronotropic response has been implicated in the reduction in aerobic capacity.

The results of this study indicate that decreased aerobic capacity is not directly related to the heart rate response. The poor correlation between peak heart rate and $\mathrm{VO}_{2}$ peak $(r=0.07, P=.76)$ in this study suggests that, at least over the range of chronotropic responses observed in these patients, $\mathrm{VO}_{2}$ peak depends more on other factors than on the heart rate response alone. Some of these other factors may include widening of the arteriovenous oxygen difference or changes in stroke volume. Although the level of regular physical activity was not assessed in these patients before the study, no patients had medically prescribed exercise restrictions. Nevertheless, physical inactivity leading to aerobic deconditioning may be a reasonable speculation based on our data.

\section{Resting Pulmonary Function}

Indices of resting lung mechanics in patients evaluated in this study were mildly decreased when compared with normative data in healthy populations. The proportionate decreases in FVC and $\mathrm{FEV}_{1}$ reflect a pattern of restrictive airway physiology. This was suggested by the normal $\mathrm{FEV}_{1} / \mathrm{FVC}$ ratio observed in this group. However, proportionally greater reductions in mean expiratory flows $\left(\mathrm{FEF}_{25-75}\right)$ were observed, suggesting small airway dysfunction commonly seen in obstructive airway physiology. Of the measurements of resting lung mechanics, $\mathrm{FEV}_{1}$ and $\mathrm{FEV}_{1} / \mathrm{FVC}$ (Figure E1) were independently related to $\mathrm{VO}_{2}$ peak. There- 
fore, data from this study suggest an obstructive component of airway physiology such that when coupled to an underlying mild restrictive process, a more profound impact on aerobic capacity is observed.

Residual pulmonary abnormalities are not unique to TAPVC physiology. Pulmonary abnormalities have generally been reported in cardiac lesions requiring even a single palliative procedure ${ }^{15}$ However, the findings of this study conflict with those previously reported by Paridon and colleagues, ${ }^{2}$ whose pulmonary data in patients surgically repaired for TAPVC did not reflect any component of obstructive airway physiology. Reasons for such a pattern in airway physiology have been attributed to several factors including the effect of multiple thoracic surgical interventions on chest wall mechanics, diminished blood flow resulting in chronic cyanosis, and abnormal pulmonary growth patterns in utero. ${ }^{17}$ Rationale for the former 2 factors is difficult to explain in the current study given that most of the patients underwent a single operative procedure early after birth. The latter factor may be more plausible in this cohort given the persistent pulmonary abnormalities despite early repair. However, the exact mechanism has yet to be fully elucidated.

In a single study performed at the same institution, Mahle and colleagues ${ }^{18}$ examined pulmonary and cardiovascular function at rest and during exercise in a subset $(\mathrm{n}=22)$ of children (mean age $=11$ years) surgically repaired for D-transposition of the great arteries during infancy. All patients in their study underwent a single surgical repair using similar perioperative techniques (eg, cardiopulmonary bypass, deep hypothermic circulatory arrest). Identical protocols for the measurements of pulmonary mechanics at rest and cardiovascular performance during exercise were used. Although the surgical intervention used to palliate D-transposition of the great arteries is arguably more complex than that of TAPVC, Mahle and colleagues reported mean values for pulmonary function at rest and cardiovascular performance during exercise were $100 \%$ and $113 \%$ of predicted for age and gender, respectively. Therefore, data from the current study implicate in utero developmental changes unique to TAPVC physiology as more likely rationale for the persistent abnormalities in pulmonary function and exercise performance rather than the effect of thoracic surgery.

\section{Exercise Pulmonary Function}

Data from this study indicate that the dynamic responses of the pulmonary system to exercise in these children surgically repaired for TAPVC during infancy are different than those of children of similar age and gender. Table 4 illustrates the increase in VE and the relative contribution of its components ("f" and TV) in response to progressive exercise. Similarly, the increase in TV/FVC and VE/ $/ \mathrm{VO}_{2}$ illus- trates the relative increase in depth of breathing and increasing ventilation in relation to $\mathrm{VO}_{2}$, respectively.

It has been suggested that children have breathing patterns similar to that in adults, as measured by the relative contributions of " $\mathrm{f}$ " and TV to VE during progressive exercise. In 177 boys and girls (mean age $=11$ years), Armstrong and colleagues ${ }^{19}$ showed as exercise increased to maximal levels, there was a greater reliance on " $\mathrm{f}$ " to create $\mathrm{VE}$ as indicated by the increase in f/TV ratio $(41 \%-47 \%)$, whereas the TV/FVC ratio remained relatively stable (38\%$41 \%$ ) throughout progressive exercise. Data from the current study are consistent with such a pattern. However, the extent to which the TV could increase was limited, as noted by the lower average value for TV across exercise intensities. Therefore, the greater than normal increase in " $\mathrm{f}$ " to meet the ventilatory requirement for exercise likely contributed to the high DS/TV seen at peak exercise as a consequence of greater ventilation of anatomic dead space rather than any intrinsic lung parenchymal disease.

The analysis of the slopes of $\mathrm{VE}$ versus $\mathrm{VCO}_{2}$ enabled an assessment of the breathing patterns related to the efficiency of the ventilatory response to metabolic demands. Data from this study indicate $72 \%$ of the patients had abnormal ventilatory responses to progressive exercise when compared with normal values for age and gender. Although these results are superior to data reported in other congenital cardiac lesions, residual hemodynamic abnormalities often seen in such defects as tetralogy of Fallot and Fontan physiology preclude reliable comparison. ${ }^{17,20}$

Regardless of the cause of the pulmonary impairment, exercise performance in children surgically repaired for congenital heart disease is seldom limited by their pulmonary system. ${ }^{21}$ This is because the impaired function of the cardiovascular system usually limits oxygen delivery before the pulmonary reserve is exhausted. The adequate BR at peak exercise $(19 \% \pm 11 \%)$ in this study suggests the limitation in exercise performance was not primarily a factor of their underlying pulmonary abnormalities.

\section{Neurologic Function}

Neurologic status and exercise performance have been assessed in a variety of congenital heart anomalies requiring early surgical repair. However, the relationship of one to the other has not been explored. To the extent that neurologic function was assessed, the results of this study are inconclusive. Although these results indicate that a subgroup of children surgically repaired for TAPVC during infancy exhibit a pattern of neurologic dysfunction characterized by deficits in attention, hyperactivity, and neuromuscular function, these abnormalities do not clearly affect late cardiopulmonary exercise performance. This is encouraging given the persistent neurodevelopmental abnormalities seen in 
other congenital heart defects requiring early surgical repair. ${ }^{22,23}$

\section{Limitations}

The main limitation of this study was the cross-sectional design. Therefore, inferences about the long-term impact of surgical repair for TAPVC during infancy are limited. This study was also limited by its small sample size. On the basis of the similarities between those who returned for testing and those who were eligible but did not return, there does not seem to be an obvious selection bias. However, other factors not accounted for between those subjects who returned for testing and those who optioned not to return, such as parental status, race and ethnicity, and geographic demographics, could contribute to the results of this study. In addition, the relative small size of the sample precluded more sophisticated statistical modeling and analysis. Finally, the noninvasive assessment of exercise performance may have precluded the identification of all cardiovascular, pulmonary, and hemodynamic abnormalities otherwise identified with more invasive methods.

\section{Conclusions}

Although the subjects were clinically asymptomatic at rest, residual abnormalities of the cardiovascular and pulmonary system during exercise persist at late follow-up in children surgically repaired for TAPVC during infancy. Specifically, obstructive airway physiology is evident on long-term follow-up. Whether this attribute is unique to abnormal development of the pulmonary veins or is evidence of subclinical pulmonary disease has yet to be fully clarified. As in other congenital heart defects requiring early surgical repair, deficits in neurologic function persist at late follow-up after repair for TAPVC during infancy. However, the impact on long-term functional outcome, as measured by the indices of exercise performance, has yet to be fully clarified. Although the mild abnormalities in cardiovascular, pulmonary, and neurologic function seem to be well tolerated, careful continued observation is required as these patients enter adulthood.

\section{References}

1. Bando K, Turrentine MW, Esing GJ, Sun K, Sharp TG, Sekine Y. Surgical management of total anomalous pulmonary venous connection. Thirty-year trends. Circulation. 1996;94:II12-6.

2. Paridon SM, Sullivan NM, Schneider J, Pinsky WW. Cardiopulmonary performance at rest and exercise following repair of total anomalous pulmonary venous connection. Am J Cardiol. 1993;72:1444-7.

3. Kirshbom PM, Myung RJ, Gaynor JW, Ittenbach RF, Paridon SM, DeCampli WM, et al. Preoperative pulmonary venous obstruction affects long-term outcome for survivors of total anomalous pulmonary venous connection repair. Ann Thorac Surg. 2002;74:1616-20.

4. American Thoracic Society. Standardization of spirometry, 1994 update. Am J Res Crit Care Med. 1995;152:1107-36.

5. Hankinson JL, Odencrantz JR, Fedan KB. Spirometric reference values from a sample of the general U.S. population. Am J Respir Crit Care Med. 1999;159:179-87.

6. Wasserman K, Hansen JE, Sue DY, Casaburi R, Whipp BJ. Physiology of exercise. In: Weingberg R, editor. Principals of Exercise Testing and Interpretation. 3rd ed. Philadelphia, PA: Lippincott Williams \& Wilkins; 1999:10-59.

7. Rowland T. Aerobic exercise testing protocols. In: Rowland R, editor. Pediatric Laboratory Exercise Testing. Champaign, IL: Human Kinetics; 1993:29-31.

8. Paul MH, Wessel HU. Exercise studies in patients with transposition of the great arteries after atrial repair operations (Mustard/Senning): a review. Pediatr Cardiol. 1999;20:49-55.

9. Cooper DM, Weiler-Ravell D, Whipp BJ, Wasserman K. Aerobic parameters of exercise as a function of body size during growth in children. J Appl Physiol. 1984;56:628-34.

10. Beaver WL, Wasserman K, Whipp BJ. A new method for detecting the anaerobic threshold during exercise and recovery in man. $J$ Appl Physiol. 1986;60:2020-7.

11. Whipp BJ, Wasserman K. Alveolar-arterial gas tension differences during graded exercise. J Appl Physiol. 1969;27:361-5.

12. Cooper DM, Kaplan MR, Baumgarten L, Weiler-Ravell D, Whipp BJ, Wasserman $\mathrm{K}$. Coupling of ventilation and $\mathrm{CO}_{2}$ production during exercise in children. Pediatr Res. 1987;21:568-72.

13. Kaltman JR, Jarvik GP, Bernbaum J, Wernovsky G, Gerdes M, Zackai E, et al. Neurodevelopmental outcome after early repair of a ventricular septal defect with or without aortic arch obstruction. J Thorac Cardiovasc Surg. 2006;131:792-8.

14. Kirshbom PM, Flynn TB, Clancy RR, Ittenbach RF, Hartman DM, Paridon SM, et al. Late neurodevelopmental outcome after repair of total anomalous pulmonary venous connection. $J$ Thorac Cardiovasc Surg. 2005;129:1091-7.

15. Mahle WT, McBride MG, Paridon SM. Exercise performance in tetralogy of Fallot: the impact of primary complete repair in infancy. Pediatr Cardiol. 2002;23:224-9.

16. Byrum CJ, Dick M, Behrendt DM, Rosenthal A. Repair of total anomalous pulmonary venous connection in patients younger than 6 months old. Late postoperative hemodynamic and electrophysiologic status. Circulation. 1982;66:208-14.

17. Reybrouck T, Boshoff D, Vanhees L, Defoor J, Gewillig M. Ventilatory response to exercise in patients after correction of cyanotic congenital heart disease: relation with clinical outcome after surgery. Heart. 2005;90:215-6.

18. Mahle WT, McBride MG, Paridon SM. Exercise performance following the arterial switch operation for D-transposition of the great arteries. Am J Cardiol. 2001;87:753-8.

19. Armstrong N, Kirby BJ, McManus AM, Welsman JR. Prepubescents' ventilatory responses to exercise with reference to sex and body size. Chest. 1997;112:1554-60.

20. Rowe SA, Zahka KG, Manolio TA, Horneffer PJ, Kidd L. Lung function and pulmonary regurgitation limit exercise capacity in postoperative tetralogy of Fallot. J Am Coll Cardiol. 1991;17:461-6.

21. Paridon SM. Congenital heart disease: cardiac performance and adaptations to exercise. Pediatr Exerc Sci. 1997;9:308-23.

22. Bellinger DC, Wypij D, duDuplessis AJ, Rappaport LA, Jonas RA, Wernovsky G, et al. Neurodevelopmental status at eight years in children with dextro-transposition of the great arteries: The Boston Circulatory Arrest Trial. J Thorac Cardiovasc Surg. 2003;126:1385-96.

23. Wernovsky G, Stiles KM, Gauvreau K, Gentles TL, duPlessis AJ, Bellinger DC, et al. Cognitive development after the Fontan operation. Circulation. 2000;102:883-9. 


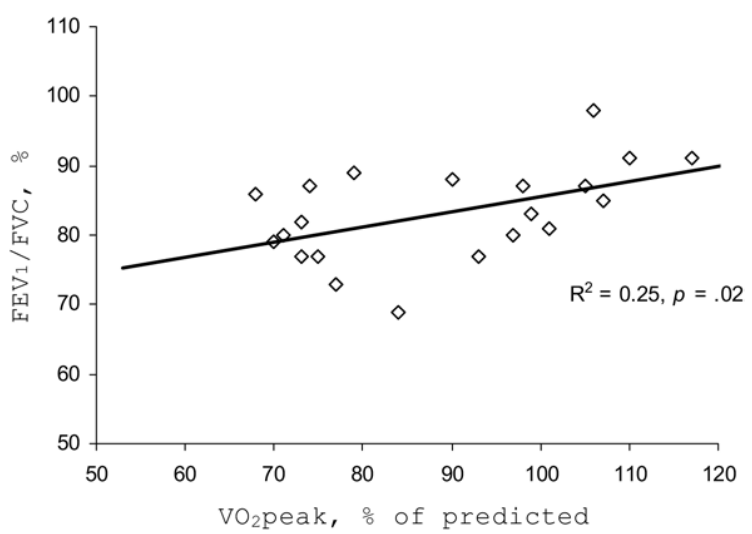

Figure E1. Relationship of $F E V_{1} / F V C$ to peak oxygen consumption. $F E V_{1}$, Forced expiratory volume in 1 second; $F V C$, forced vital capacity; $\mathrm{VO}_{2}$, minute oxygen consumption.
TABLE E1. Demographic and echocardiographic characteristics of subjects at follow-up

\begin{tabular}{lc}
\hline Variable & Value \\
\hline Demographics & \\
Age at testing, y & $11.0 \pm 3.6$ \\
Height, cm & $143.8 \pm 17.6$ \\
Weight, $\mathrm{kg}$ & $41.0 \pm 16.4$ \\
BSA, m & $1.2 \pm 0.3$ \\
Echocardiography & \\
RV pressure, mm Hg & $23.1 \pm 6.1$ \\
LVSF, \% & $36.0 \pm 3.6$ \\
Pulmonary venous obstruction & $1(4)$ \\
\hline
\end{tabular}

$B S A$, Body surface area; $R V$, right ventricular; $L V S F$, left ventricular shortening fraction. Values represent mean \pm standard deviation, or in the case of pulmonary venous obstruction, number of observations and percentage of total population $(\%)$

TABLE E2. Neurologic examination

\begin{tabular}{llcc}
\hline Variable & Normal & Abnormal & Suspect \\
\hline Hyperactivity & $20(74 \%)$ & $7(26 \%)$ & - \\
Inattentiveness & $15(56 \%)$ & $12(44 \%)$ & - \\
Fine motor function & $20(74 \%)$ & $7(26 \%)$ & - \\
Gross motor function & $25(93 \%)$ & $2(7 \%)$ & - \\
Overall neuromuscular assessment & $20(74 \%)$ & $2(7 \%)$ & $5(19 \%)$ \\
Overall neurologic assessment & $17(63 \%)$ & $6(22 \%)$ & $4(15 \%)$ \\
\hline
\end{tabular}

Data are represented as number of observations and percentage of total population. Overall neuromuscular assessment: composite score for fine and gross motor function. Overall neurologic assessment: composite score for all four domains (hyperactivity, inattentiveness, fine motor, gross motor). 
TABLE E3. Multiple covariate models

\begin{tabular}{|c|c|c|c|c|c|c|}
\hline Covariate & $\beta$-coefficient & SE & $t$ & $P$ & Model $\boldsymbol{P}$ value & $\overline{R^{2}}$ \\
\hline $\mathrm{VO}_{2}$ peak model* & & & & & .02 & .33 \\
\hline $\mathrm{FEV}_{1}$ & .38 & .21 & 1.01 & .07 & & \\
\hline $\mathrm{O}_{2} \mathrm{P}$ & .35 & .17 & 1.96 & .06 & & \\
\hline Intercept & 21.83 & 21.72 & 1.01 & & & \\
\hline VAT modelt & & & & & .07 & .31 \\
\hline PDBP & 1.10 & .07 & .34 & .08 & & \\
\hline Composite Neuromuscular Score & 12.78 & 11.92 & 1.07 & .11 & & \\
\hline Intercept & 13.49 & 39.49 & .34 & & & \\
\hline PWC model ${ }^{*}$ & & & & & .01 & .36 \\
\hline Gender & 18.23 & 9.28 & 1.96 & .06 & & \\
\hline $\mathrm{O}_{2} \mathrm{P}$ & 3.24 & 1.37 & 2.37 & .03 & & \\
\hline Intercept & 64.90 & 12.88 & 5.04 & & & \\
\hline
\end{tabular}

$V O_{2}$, Minute oxygen consumption; $S E$, standard error; $F E V_{1}$, forced expiratory volume in 1 second; $O_{2} P$, oxygen pulse; $V A T$, ventilatory anaerobic threshold; $P D B P$, peak diastolic blood pressure; $P W C$, physical working capacity. Composite neuromuscular score: fine and gross motor assessment. ${ }^{*} n=22$. $t n=25$. 